\section{JTI}

JOURNAL OF

TRAUMA AND INJURY

Received: May 11, 2020

Revised: August 26, 2020

Accepted: October 6, 2020

\section{Correspondence to}

Jae Guk Kim, M.D.

Department of Neurology, Daejeon Eulji Medical Center, Eulji University, 95 Dun-

sanseo-ro, Seo-gu, Daejeon 35233, Korea

Tel: $+82-42-611-3429$

Fax: $+82-42-611-3858$

E-mail: jgkim@eulji.ac.kr

\title{
Cerebral Fat Embolism That Was Initially Negative on Diffusion- Weighted Magnetic Resonance Imaging
}

\author{
Seung Je Go, M.D. ${ }^{1}$, Yun Su Mun, M.D. ${ }^{1}$, Seung Ho Bang, M.D. ${ }^{1}$, \\ Yong Han Cha, M.D., Ph.D. ${ }^{2}$, Young Hoon Sul, M.D., Ph.D. ${ }^{3}$, Jin Bong Ye, M.D. ${ }^{3}$, \\ Jae Guk Kim, M.D. ${ }^{4}$ \\ ${ }^{1}$ Department of Trauma Surgery, Trauma Center of Daejeon Eulji Medical Center, \\ Eulji University, Daejeon, Korea \\ ${ }^{2}$ Department of Orthopedic Surgery, Trauma Center of Daejeon Eulji Medical Center, \\ Eulji University, Daejeon, Korea \\ ${ }^{3}$ Department of Trauma Surgery, Trauma Center of Chungbuk National University \\ Hospital, Cheongju, Korea \\ ${ }^{4}$ Department of Neurology, Daejeon Eulji Medical Center, Eulji University, Daejeon, Korea
}

Fat embolism syndrome is a rare, but serious condition that occurs in patients with fractures of the long bones or who undergo orthopedic surgery. The main clinical features of fat embolism syndrome are an altered mental status, hypoxia, and petechial rash. Cerebral fat embolism is the most severe manifestation of fat embolism syndrome because it can lead to an altered mental status. The diagnosis of cerebral fat embolism is clinical, but brain magnetic resonance image (MRI) is helpful. There is usually an interval until symptoms, such as an altered mental status, develop after trauma. We report a case of cerebral fat embolism in which the patient's mental status deteriorated several hours after trauma and the initial findings were negative on diffusion-weighted MRI.

Keywords: Embolism, fat; Magnetic resonance

\section{INTRODUCTION}

Fat embolism syndrome (FES) is an uncommon, but potentially life-threatening condition occurring in patients with fractures of the long bones or pelvis. The clinical 
manifestations of FES include neurological symptoms, hypoxia, and gradual development of a petechial rash after trauma [1]. Among these manifestations, cerebral fat embolism (CFE) is particularly concerning because it can lead to an altered mental status. CFE is a clinical diagnosis, but specific findings on neuroimaging studies can be strongly supportive. Brain computed tomography (CT) is not diagnostic for CFE, while brain magnetic resonance imaging (MRI) is more sensitive. However, initial diffusion-weighted imaging (DWI) on brain MRI may be negative because there is usually an interval until the symptoms develop after trauma. Herein, we report a case of CFE in which the patient's mental status deteriorated within several hours after trauma and had initially negative DWI.

\section{CASE REPORT}

A 59-year-old man visited the emergency room after a motorcycle accident. On arrival, he was alert and oriented without external evidence of head trauma. He complained of pain in the pelvic area and both thighs. The vital signs were stable and the results of the initial blood tests, including arterial blood gas analysis, were normal. Brain and abdominal CT scans revealed no abnormalities. Simple X-rays showed an inter-trochanteric fracture of the right femur and a distal shaft fracture of the left femur (Fig. 1).

While additional X-rays were being taken, the patient abruptly became stuporous and developed hypoxemia,
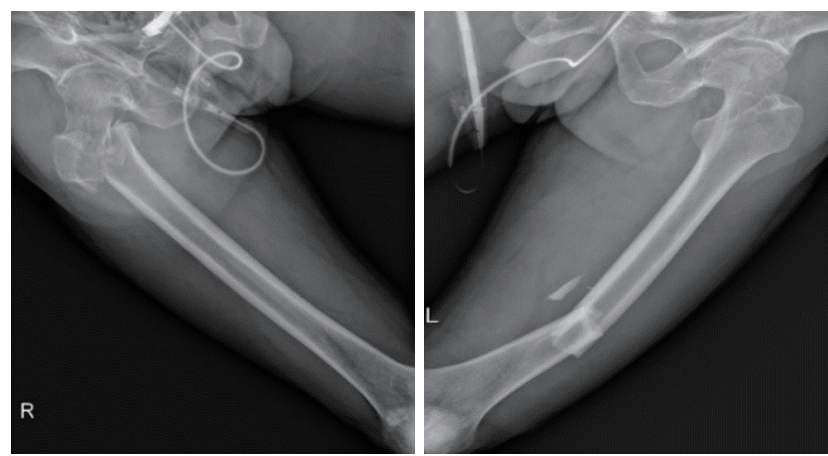

Fig. 1. X-rays show fractures of the right femur intertrochanter and the left femur shaft. requiring endotracheal intubation and mechanical ventilation. A repeated brain CT scan showed no abnormal findings, and brain DWI also demonstrated no abnormalities (Fig. 2). On the second day, chest CT showed no definite evidence of pulmonary thromboembolism and echocardiography showed no abnormalities in regional wall motion and valvular function. On the third day, a neurologist was consulted regarding the patient's diminished mental status. On a neurological examination, the patient had roving eye movements and decerebrate posturing. Electroencephalography was done to exclude non-convulsive status epilepticus, but there was no epileptiform discharge. Follow-up brain MRI was conducted, and there were innumerable punctate foci of restricted diffusion, producing a "starfield" appearance on DWI (Fig. 3). After the diagnosis of CFE, he received steroid treatment, but his mental status did not change. Neuropsychological testing was not possible because of mutism. After a month with no change of consciousness, he opened his eyes spontaneously, but could not make comprehensible sounds. Follow-up MRI performed 40 days after trauma showed slight resolution of the previous multifocal fat embolism and interval development of hypoxic-ischemic insults. He was discharged to another hospital for rehabilitation with no change of consciousness.

\section{DISCUSSION}

FES is an uncommon, but potentially lethal condition that occurs in patients with long bone and pelvic fractures. FES, which was first described by Zenker in 1861, is characterized by major features such as altered mental status, hypoxemia, and petechial rash, as well as minor features such as tachycardia, pyrexia, retinal petechia, a sudden drop in hemoglobin levels, sudden thrombocytopenia, oliguria, a high erythrocyte sedimentation rate, and fat globules in the sputum [2]. The most severe manifestation of FES is CFE, which can lead to an altered mental status on a spectrum from confusion to coma. The clinical symptoms of CFE usually develop gradually within 24 to 72 hours after trauma [3]. Hypoxemia can lead to the requirement for endotracheal intubation and mechanical ventilator use. A petechial rash is observed in the con- 


\section{JTI}

junctiva, around the neck, the axillary area, and the upper body in $20-50 \%$ of FES patients [4]. In our case, the decline of the patient's consciousness first occurred and deteriorated rapidly within a few hours after trauma, and then his mental status became comatose. We performed endotracheal intubation and applied a mechanical ventilator because of the hypoxemia.

The pathophysiology of CFE involves high pressure in the marrow due to fracture or orthopedic surgery forcing marrow fat into the veins. The disruption of marrow-containing long bone allows globules of marrow fat to pass into the venous sinuses that drain the marrow and then into the systemic circulation [5]. Having reached the circulation, fat may cause symptoms as a result of embolic occlusion of arteries in the lung, brain, skin, and elsewhere [6].

CFE occurs after fat emboli enter the arterial circulation. Fat globules may enter the arterial circulation by two mechanisms. First, fat globules can enter the left atrium directly from the right heart through a shunt, such as a patent foramen ovale (PFO). Second, microglobules of fat may filter directly through the lung capillaries to reach the arterial system. These microemboli are small and malleable and may not lead to significant pulmonary injury. There is direct evidence of the passage of fat through a PFO, yet the absence of PFO in many patients with CFE supports the latter mechanism in some patients [7]. Therefore, PFO should be considered an additional risk factor for CFE, but is not necessary for CFE to occur [6]. In our case, echocardiography demonstrated no intra-cardiac shunt.

CFE is a clinical diagnosis, but specific findings on neuroimaging studies can be strongly supportive. Brain CT shows generally normal findings in most cases, but brain MRI is more sensitive. The most distinctive brain MRI finding is the starfield pattern, with scattered foci of high-intensity restricted diffusion on DWI. This is most apparent in the acute phase, from 4 hours to the first few
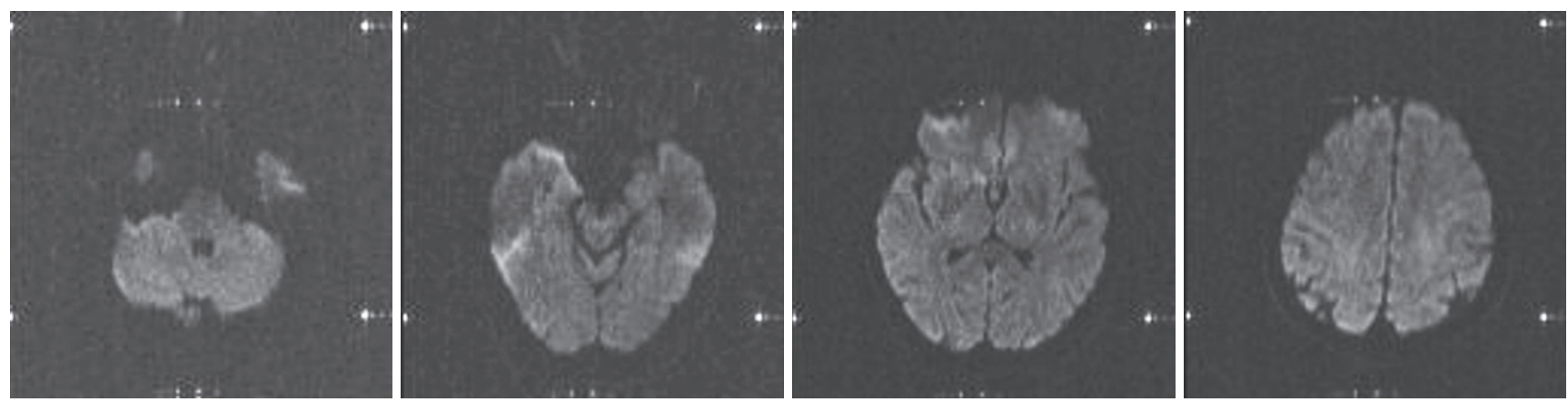

Fig. 2. Initial magnetic resonance diffusion-weighted images 3 hours after trauma display no abnormal findings.
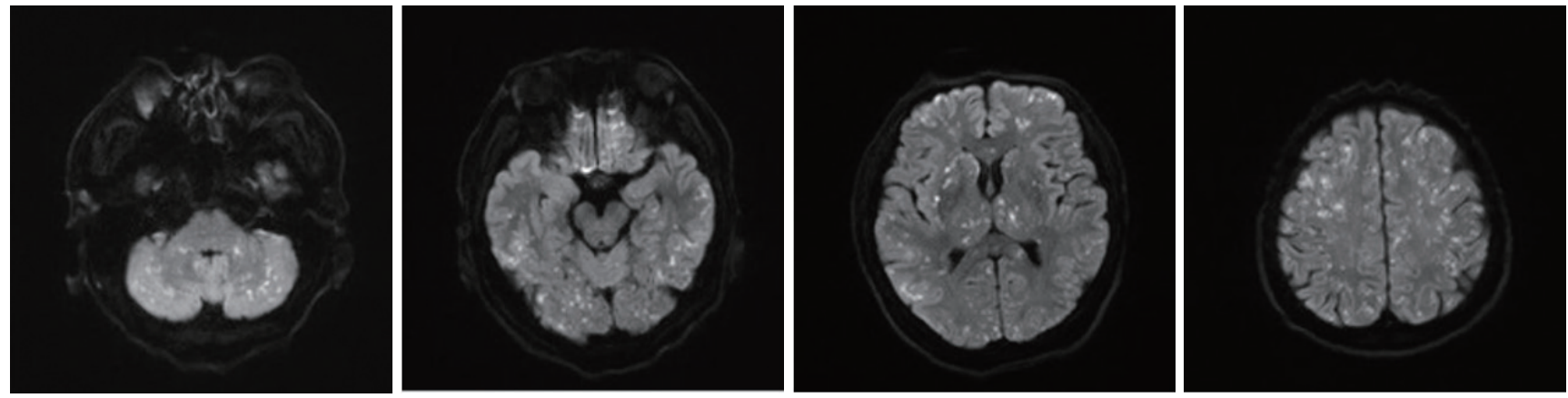

Fig. 3. Follow-up magnetic resonance diffusion-weighted images on the third day after injury demonstrate multiple hyperintense dots in the bilateral cerebellum; both hemispheres; the cerebellar peduncle; pons; both basal ganglia; both thalami; both hippocampi; both frontal, temporal, occipital, and parietal cortices; and white matter area. 
days from the time of injury [8]. However, we did not observe any abnormalities on initial DWI, which was done immediately after the patient's change in consciousness. The diffusion reflects intracellular edema due to the failure of ionic $\mathrm{Na}^{+} / \mathrm{K}^{+}$pumps. We believe that the initial negative findings on DWI may be explained by the lack of sufficient time for DWI to show intracellular edema after CFE.

There is no standard treatment for CFE. It is important to perform conservative treatment for the clinical symptoms of CFE. At first, maintaining airway and supplying oxygen are important to ensure oxygenation in tissues. Endotracheal intubation and mechanical ventilation are required for appropriate oxygenation. It is also important to maintain an effective vascular volume for sufficient blood flow to each tissue. In addition, it has been reported that early administration of a high-dose corticosteroid reduces the development of hypoxemia and fat embolism. Gupta et al. [3] proposed a regimen of methylprednisolone $(1.5 \mathrm{mg} / \mathrm{kg}$, intravenous) every 8 hours for six doses in a select group of patients with long bone or pelvic fractures at high risk of developing CFE without significant contraindications. Corticosteroids have been extensively studied with variable results, but their use is still controversial. We treated the patient with methylprednisolone ( $1 \mathrm{~g} /$ day) for 5 days, applying the usual regimen used in steroid mega-dose therapy. We also administered enough crystalloid fluid and supplemented with albumin (if albumin $<3.0 \mathrm{~g} /$ $\mathrm{dL}$ ) while checking the patient's urine output to maintain the intra-vascular volume. However, these steps were not helpful for recovery of cognition.

The prognosis of FES has been reported to be relatively good. Therefore, most patients with FES improve relative to their initial clinical status. However, some patients with FES have been reported to have a mortality risk of 5-20\% [9]. Especially, the patients with brain MRI findings such as extensive involvement of the cerebellum and more pronounced existing lesions on follow-up observations have a poor prognosis. Takahashi et al. [8] found that the size and number of points in the starfield pattern on brain MRI obtained during the hospital course were closely correlated with the Glasgow Coma Scale at the onset of CFE. They demonstrated that widespread high-intensity areas on T2-weighted MRI in the deep white matter disappeared completely in cases with good outcomes. In our case, we observed a large number of points in the starfield pattern on serial follow-up brain MRI and the patient had a poor outcome. The deterioration of cognition remained remarkable even after 1 year.

We report a case of CFE in which the patient's mental status deteriorated within several hours after trauma and had initially negative findings on diffusion-weighted MRI. Therefore, CFE should be considered in the differential diagnosis for patients who show rapid-onset coma after trauma, even if initial DWI is negative.

\section{REFERENCES}

1. Sevitt S. The significance and pathology of fat embolism. Ann Clin Res 1977;9:173-80.

2. Gregorakos L, Sakayianni K, Hroni D, Harizopoulou V, Markou $\mathrm{N}$, Georgiadou F, et al. Prolonged coma due to cerebral fat embolism: report of two cases. J Accid Emerg Med 2000;17:144-6.

3. Gupta A, Reilly CS. Fat embolism. Continuing Education in Anaesthesia Critical Care \& Pain 2007;7:148-151.

4. Shaikh N. Emergency management of fat embolism syndrome. J Emerg Trauma Shock 2009;2:29-33.

5. Mellor A, Soni N. Fat embolism. Anaesthesia 2001;56:145-54.

6. Mijalski C, Lovett A, Mahajan R, Sundararajan S, Silverman S, Feske S. Cerebral fat embolism: a case of rapid-onset coma. Stroke 2015;46:e251-3.

7. Pell AC, Hughes D, Keating J, Christie J, Busuttil A, Sutherland GR. Brief report: fulminating fat embolism syndrome caused by paradoxical embolism through a patent foramen ovale. N Engl J Med 1993;329:926-9.

8. Takahashi M, Suzuki R, Osakabe Y, Asai JI, Miyo T, Nagashima $\mathrm{G}$, et al. Magnetic resonance imaging findings in cerebral fat embolism: correlation with clinical manifestations. J Trauma 1999;46:324-7.

9. Talbot M, Schemitsch EH. Fat embolism syndrome: history, definition, epidemiology. Injury 2006;37 Suppl 4:S3-7. 